\title{
The Surgical Procedure Assessment (SPA) score predicts intensive care unit length of stay after cardiac surgery
}

\author{
Gebhard Wagener, MD, ${ }^{\text {a }}$ Moury Minhaz, BS, ${ }^{a}$ Shuang Wang, PhD, ${ }^{\mathrm{b}}$ Oliver Panzer, MD, ${ }^{\mathrm{a}}$ \\ Hannah Wunsch, MD, MSc, ${ }^{a}$ Hugh R. Playford, MBBS, MHA, FANZCA, FJFICM, ${ }^{\mathrm{c}}$ and \\ Robert N. Sladen, MBChB, MRCP(UK), FRCP $(C)^{a}$
}

\begin{abstract}
Objective: The ability to predict intensive care unit length of stay greatly facilitates triage and resource allocation for postoperative cardiac surgical patients in the intensive care unit. We developed a simple, intuitive Surgical Procedure Assessment score that integrates surgical complexity (1, low; 2, intermediate; 3, high) with patient comorbidity (A, minimal; B, substantial). We hypothesized that the Surgical Procedure Assessment score would predict intensive care unit length of stay, discriminate preoperatively between fast-track and prolongedstay patients, and compare favorably with more complex risk scores.
\end{abstract}

Methods: After institutional review board approval, 1201 cardiac surgical patients were preoperatively assigned a Surgical Procedure Assessment score, as well as a Parsonnet, Tuman, Tu, and Cardiac Anesthesia Risk Evaluation score. We compared these scores with regard to prediction of intensive care unit length of stay, as well as their concordance in predicting intensive care unit length of stay of less than 48 hours (fast track) and more than 7 days (prolonged stay).

Results: Intensive care unit length of stay increased significantly with increasing Surgical Procedure Assessment scores $(P<.01$, Cuzick's test for trend). The lowest Surgical Procedure Assessment score (1A) predicted intensive care unit length of stay of less than 48 hours, and the higher Surgical Procedure Assessment scores (2B or 3) predicted intensive care unit length of stay of more than 7 days more accurately than the Parsonnet, Tuman, Tu and Cardiac Anesthesia Risk Evaluation scores.

Conclusions: The Surgical Procedure Assessment score predicts intensive care unit length of stay better than other comparable scores. It is simple, intuitive, and easily understood by all caregivers and can preoperatively discriminate fast-track from prolonged-stay patients. It is a useful tool to facilitate intensive care unit triage. (J Thorac Cardiovasc Surg 2011;142:443-50)

The lack of readily available postoperative intensive care unit (ICU) beds is an important rate-limiting step in cardiac surgical throughput. This problem has been addressed to some extent by the development of fast-track protocols that minimize ICU time or even allow the ICU to be bypassed altogether. However, this recourse is attenuated by the national trend toward increasingly complex procedures in patients of increasingly advanced age. ${ }^{1}$ Patients with extended ICU length of stay (LOS) provide an enormous resource burden. We have previously observed that although only $4 \%$ of patients after cardiac surgery stay in the ICU

\footnotetext{
From the Department of Anesthesiology, ${ }^{a}$ College of Physicians and Surgeons, Columbia University, New York, NY; the Department of Biostatistics, ${ }^{\text {b }}$ Mailman School of Public Heath, Columbia University, New York, NY; and the Cardiothoracic Intensive Care Unit, ${ }^{\mathrm{c}}$ Westmead Hospital, Sydney, Australia.

This work was funded by intramural grant support from the Department of Anesthesiology, Columbia University College of Physicians and Surgeons, New York, NY. Disclosures: Authors have nothing to disclose with regard to commercial support. Received for publication May 28, 2010; revisions received Aug 19, 2010; accepted for publication Sept 12, 2010; available ahead of print April 18, 2011.

Address for reprints: Gebhard Wagener, MD, Department of Anesthesiology, College of Physicians \& Surgeons of Columbia University, P\&S Box 46 (PH-5), 630 West 168th St, New York, NY 10032-3784 (E-mail: gw72@columbia.edu). 0022-5223/\$36.00

Copyright (c) 2011 by The American Association for Thoracic Surgery doi:10.1016/j.jtcvs.2010.09.067
}

for more than 14 days, they account for $30 \%$ of the ICU patient days. ${ }^{2}$ Reliable preoperative prediction of postoperative ICU LOS could facilitate rational bed allocation and maximize use of the scarce resource that ICU beds represent. However, most scoring systems in present use are complex and were developed to allow cardiac surgeons and cardiologists to anticipate operative mortality and risk rather than ICU LOS and potential ICU resource use. ${ }^{3,4}$

We considered that it would be helpful to have an intuitive and simple algorithm that is easily understood by all parties involved in ICU planning: surgical case schedulers, nurses, hospital administrators, surgeons, and anesthesiologists. We therefore devised a Surgical Procedure Assessment (SPA) score based on just 2 variables: the complexity of the surgical procedure (1, low; 2 , medium; or 3 , high) and known patient comorbidity. Thus patients undergoing less complex operations (eg, coronary artery bypass grafting and single-valve operations) are assigned an SPA score of 1 , those undergoing more complex operations (eg, redo, coronary artery bypass grafting valve, and multiple-valve operations or cardiac surgery in combination with the maze procedure or heart transplantation) are assigned an SPA score of 2, and those undergoing the most complex operations (eg, ventricular assist device placement and 


\section{Abbreviations and Acronyms \\ $\mathrm{CARE}=$ Cardiac Anesthesia Risk Evaluation \\ $\mathrm{ICU}=$ intensive care unit \\ LOS = length of stay \\ SPA $=$ Surgical Procedure Assessment}

lung transplantation) are assigned an SPA score of 3. Absence of comorbidity is designated by the suffix A, and substantial comorbidity (eg, organ system failure, arrhythmias, or insulin-dependent diabetes) is designated with the suffix B (Table 1). We hypothesized that the SPA score could preoperatively discriminate between fast-track (ICU LOS $<48$ hours) and prolonged-stay (ICU LOS $>7$ days) patients and perform as well or better than 4 other validated scoring systems: the Parsonnet, ${ }^{5}$ Tuman, ${ }^{6} \mathrm{Tu},{ }^{7}$ and Cardiac Anesthesia Risk Evaluation (CARE) ${ }^{8}$ scores.

\section{MATERIALS AND METHODS}

From January 1 to December 31, 2002, all adult patients undergoing cardiac surgery at Columbia University Medical Center were enrolled in the study. The Institutional Review Board of Columbia University waived the requirement to obtain informed consent.

SPA, Parsonnet, Tuman, Tu, and CARE scores were assigned before the operation. Baseline demographics, ICU LOS, hospital LOS, and inhospital mortality were prospectively collected on all patients. The hospital charges were retrieved from the hospital billing system. Patients were designated into groups based on their ICU LOS: fast track, less than 48 hours; prolonged stay, more than 7 days.

We applied Cuzick's nonparametric test for trend, an extension of the Wilcoxon rank test, ${ }^{9}$ to evaluate whether there was a statistically significant progression of ICU LOS as the SPA score assignment increased from 1A through 3.

We compared the SPA score with the Parsonnet, Tuman, Tu, and CARE scores by developing multiple regression models that estimated the ability of each score to explain the observed variability of ICU and hospital LOS, in-hospital mortality, and hospital charges. The LOS data and hospital charges underwent logarithmic transformation to achieve a normal distribution before being entered into linear regression models. Logistic regression was used to test the association between SPA scores and 4 other validated scoring systems and in-hospital mortality. The Hosmer and Lemeshow goodness-of-fit test was applied to test model fitness with logistic regressions on in-hospital mortality. Receiver operating characteristic curves were also used to investigate the area under the curve with SPA scores and 4 other scoring systems on in-hospital mortality. Cuzick's nonparametric test for trend, an extension of the Wilcoxon rank test, was used to evaluate whether there was a statistically significant progression of ICU LOS, hospital LOS, in-hospital mortality, and hospital charges as the SPA score assignment increased from 1A through 3.

We tested the ability of all 5 scoring systems to predict fast-track and prolonged-stay patients. Relative risks, odds ratios, sensitivities, specificities, positive and negative predictive values, and likelihood ratios were used to evaluate the concordance of the lowest SPA score (1A) with ICU LOS of less than 48 hours in comparison with the lowest Parsonnet $(<5)$, Tuman $(<2), \mathrm{Tu}(<2)$, and CARE $(1)$ scores, respectively. We further tested these measures against an even shorter ICU LOS ( $<24$ hours).

The same tests were used to evaluate the concordance of the highest SPA scores (2B and 3) with ICU LOS of more than 7 days in comparison with the highest Parsonnet $(>15)$, Tuman $(>6)$, Tu $(>6)$, and CARE $(4,5$, or 5E)
TABLE 1. Classification of the Surgical Procedure Assessment score SPA

\begin{tabular}{lcc} 
score & Surgical factors & Patient factors \\
\hline 1A & Low complexity (eg, CABG, AVR, & No comorbidity \\
IB & MVR) & + Comorbidity
\end{tabular}

2A Moderate complexity (eg, CABG No comorbidity

2B and valve, multiple valves, +Comorbidity reoperation, cardiac surgery + maze procedure, heart transplantation)

3 High complexity (eg, ventricular assist device, lung transplantation)

\pm Comorbidity

Comorbidity:

- Congestive heart failure requiring inotropic support

- Milrinone, dobutamine, nesiritide, or intra-aortic balloon pump

- LV ejection fraction $<20 \%$

- Arrhythmias: ventricular tachycardia

- Liver disease

- Increased bilirubin level or coagulation parameters

- Insulin-dependent diabetes

- End-stage renal failure

- Symptomatic COPD

- Home oxygen

- Severe systemic disease (eg, systemic lupus, rheumatoid arthritis, sickle cell disease)

SPA, Surgical Procedure Assessment; $C A B G$, coronary artery bypass grafting; $A V R$, aortic valve replacement or repair; $M V R$, mitral valve replacement or repair; $L V$, left ventricular; $C O P D$, chronic obstructive pulmonary disease.

scores, respectively. We further tested these measures against an even longer ICU LOS (>10 days).

Values are presented as means \pm standard deviations. Comparisons between groups were made by using the unpaired $t$ test for values with Gaussian distribution and by using the Mann-Whitney (Wilcoxon rank) test or Spearman test for correlation for continuous variables without normal distribution. Gaussian distribution was determined by using the Kolmogorov-Smirnov test. The $\chi 2$ test or 1-way analysis of variance was used as appropriate. $P$ values were 2 -tailed.

SAS 9.1 software (SAS, Inc, Cary, NC) was used for the regression models, and SPSS 11.0.4 (SPSS, Inc, Chicago, Ill) and GraphPad Prism 4.0 (GraphPad, Inc, San Diego, Calif) software were used for remainder of the statistical analysis.

\section{RESULTS \\ Patients' Characteristics}

All 1201 adult patients undergoing cardiac surgery from January 1, 2002, to December 31, 2002, at Columbia University Medical Center were included. One thousand one hundred two $(91.2 \%)$ patients were admitted to the cardiothoracic intensive care unit, and 99 patients $(8.8 \%)$ were admitted to the surgical intensive care unit of Columbia University Medical Center. Patients' demographics and types of cardiac surgical procedures are listed in Table 2. The entire group of 1201 cardiac surgical patients used 4065 ICU bed days, with a mean ICU LOS of 3.38 days. Of these, only $106(8.8 \%)$ had a prolonged stay (ICU LOS $>7$ days), but these patients used $45.6 \%$ of all ICU bed days. 
TABLE 2. Patients' characteristics

\begin{tabular}{|c|c|c|c|c|c|c|}
\hline & $\begin{array}{c}\text { SPA 1A } \\
393(32.7 \%)\end{array}$ & $\begin{array}{c}\text { SPA 1B } \\
165(13.7 \%)\end{array}$ & $\begin{array}{c}\text { SPA 2A } \\
303(25.2 \%)\end{array}$ & $\begin{array}{c}\text { SPA 2B } \\
307(25.6 \%)\end{array}$ & $\begin{array}{c}\text { SPA 3 } \\
33(2.8 \%)\end{array}$ & $\begin{array}{c}P \text { value } \\
\text { (ANOVA) }\end{array}$ \\
\hline \multicolumn{7}{|l|}{ Preoperative } \\
\hline Female sex, n (\%) & $104(26.5)$ & $70(42.4)$ & $104(34.3)$ & $123(40.1)$ & $9(27.3)$ & .0035 \\
\hline Age $(y)$, mean $\pm S D$ & $64.2 \pm 13.0$ & $66.5 \pm 12.4$ & $63.7 \pm 15.4$ & $63.8 \pm 14.7$ & $50.3 \pm 15.9$ & $<.0001$ \\
\hline BMI $\left(\mathrm{kg} / \mathrm{m}^{2}\right)$, mean $\pm \mathrm{SD}$ & $29.6 \pm 16.9$ & $28.9 \pm 14.6$ & $29.2 \pm 20.0$ & $30.7 \pm 30.1$ & $27.5 \pm 6.2$ & .0122 \\
\hline $\begin{array}{l}\text { End-stage renal failure, } \\
\mathrm{n}(\%)\end{array}$ & 0 & $11(6.7)$ & 0 & $9(2.9)$ & $4(12.1)$ & .004 \\
\hline Diabetes, $\mathrm{n}(\%)$ & $90(22.9)$ & $57(34.5)$ & $57(18.8)$ & $85(27.7)$ & $8(24.2)$ & NS \\
\hline IDDM, n (\%) & 0 & $27(16.4)$ & 0 & $27(8.8)$ & $2(6.1)$ & NS \\
\hline NIDDM, n (\%) & $90(22.9)$ & $30(18.2)$ & $57(18.8)$ & $58(18.9)$ & $6(18.2)$ & NS \\
\hline $\begin{array}{l}\text { Ejection fraction, } \\
\text { mean } \pm \mathrm{SD}\end{array}$ & $51.7 \pm 11.4$ & $46.8 \pm 14.4$ & $51.5 \pm 12.1$ & $41.2 \pm 17.5$ & $21.2 \pm 11.4$ & $<.0001$ \\
\hline \multicolumn{7}{|l|}{ Operations } \\
\hline CABG, n (\%) & $279(71.0)$ & $88(53.3)$ & $104(34.3)^{*}$ & $55(17.9)^{*}$ & 0 & \\
\hline OPCABG, n (\%) & $91(23.2)$ & $20(12.1)$ & $19(6.3)^{*}$ & $13(4.2)^{*}$ & 0 & \\
\hline Single AVR, n (\%) & $68(17.3)$ & $28(17.0)$ & $28(9.2)^{*}$ & $24(7.8)^{*}$ & 0 & \\
\hline Single MVR, n (\%) & $42(10.7)$ & $43(26.1)$ & $15(5.0)^{*}$ & $18(5.9)^{*}$ & 0 & \\
\hline Other single valves, $\mathrm{n}(\%)$ & $1(0.3)$ & $4(2.4)$ & 0 & $2(0.7)$ & 0 & \\
\hline Multiple valves , n (\%) & 0 & 0 & $12(4.0)$ & $48(15.6)$ & 0 & \\
\hline CABG and valve, $\mathrm{n}(\%)$ & 0 & 0 & $81(26.7)$ & $71(23.1)$ & 0 & \\
\hline VAD, n $(\%)$ & 0 & 0 & 0 & 0 & $33(100)$ & \\
\hline $\begin{array}{l}\text { Heart transplantation, } \\
\mathrm{n}(\%)\end{array}$ & 0 & 0 & $3(1.0)$ & $68(22.1)$ & 0 & \\
\hline Reoperation, n (\%) & 0 & 0 & $76(25.1)$ & $85(27.7)$ & $12(36.4)$ & $<.0001$ \\
\hline Other, n $(\%)$ & $3(0.8)$ & $2(1.2)$ & $60(19.8)$ & $21(6.8)$ & 0 & $<.0001$ \\
\hline \multicolumn{7}{|l|}{ Intraoperative } \\
\hline $\begin{array}{l}\text { CPB time (h:min), } \\
\text { mean } \pm \mathrm{SD}\end{array}$ & $1: 18 \pm 0: 51$ & $1: 38 \pm 0: 53$ & $2: 26 \pm 5: 48$ & $2: 19 \pm 1: 08$ & $1: 56 \pm 1: 05$ & $<0.0001$ \\
\hline $\begin{array}{l}\text { AXC time (h:min), } \\
\text { mean } \pm \mathrm{SD}\end{array}$ & $0: 51 \pm 0: 36$ & $1: 05 \pm 0: 37$ & $1: 14 \pm 0: 47$ & $1: 32 \pm 0: 47$ & $0: 29 \pm 0: 33$ & $<.0001$ \\
\hline \multicolumn{7}{|l|}{ Postoperative } \\
\hline ICU LOS (d), mean \pm SD & $1.6 \pm 1.5$ & $2.3 \pm 2.5$ & $3.1 \pm 5.4$ & $5.4 \pm 7.8$ & $13.8 \pm 12.9$ & $<.0001$ \\
\hline $\begin{array}{l}\text { Hospital LOS }(\mathrm{d}) \\
\text { mean } \pm \text { SD }\end{array}$ & $6.7 \pm 5.6$ & $7.8 \pm 4.6$ & $9.2 \pm 10.0$ & $16.1 \pm 19.7$ & $46.0 \pm 36.1$ & $<.0001$ \\
\hline $\begin{array}{c}\text { Charges/US dollars, } \\
\text { mean } \pm \mathrm{SD}\end{array}$ & $56,693 \pm 37,912$ & $68,997 \pm 42,474$ & $88,971 \pm 102,127$ & $200,083 \pm 251,684$ & $510,068 \pm 286,866$ & $<.0001$ \\
\hline ICU mortality, n (\%) & $1(0.3)$ & $3(1.8)$ & $10(3.3)$ & $19(6.2)$ & $6(18.2)$ & $<.0001$ \\
\hline Hospital mortality, n (\%) & $1(0.3)$ & $4(2.4)$ & $10(3.3)$ & $26(8.5)$ & $9(27.3)$ & $<.0001$ \\
\hline \multicolumn{7}{|l|}{ Scores } \\
\hline Parsonnet, mean \pm SD & $9.9 \pm 7.7$ & $16.3 \pm 9.5$ & $15.0 \pm 10.4$ & $21.6 \pm 12.6$ & $23.7 \pm 15.0$ & $<.0001$ \\
\hline CARE score, mean $\pm \mathrm{SD}$ & $1.8 \pm 0.6$ & $2.4 \pm 0.7$ & $3.2 \pm 1.1$ & $4.1 \pm 1.0$ & $4.4 \pm 1.1$ & $<.0001$ \\
\hline Tuman score, mean \pm SD & $2.2 \pm 1.8$ & $3.5 \pm 1.9$ & $4.2 \pm 2.6$ & $5.3 \pm 2.7$ & $5.7 \pm 2.9$ & $<.0001$ \\
\hline Tu score, mean $\pm \mathrm{SD}$ & $2.7 \pm 1.7$ & $3.7 \pm 1.8$ & $4.1 \pm 2.6$ & $5.0 \pm 2.4$ & $4.8 \pm 2.8$ & $<.0001$ \\
\hline
\end{tabular}

$S P A$, Surgical Procedure Assessment; ANOVA, analysis of variance; $S D$, standard deviation; $B M I$, body mass index; NS, not significant ( $P>.05$ ); IDDM, insulin-dependent diabetes mellitus; NIDDM, non-insulin-dependent diabetes mellitus; $C A B G$, coronary artery bypass grafting; $O P C A B G$, off-pump coronary artery bypass grafting; $A V R$, aortic valve replacement or repair; $M V R$, mitral valve replacement or repair; $V A D$, ventricular assist device; $C P B$, cardiopulmonary bypass; $A X C$, aortic crossclamp time; $I C U$, intensive care unit; $L O S$, length of stay; CARE, Cardiac Anesthesia Risk Evaluation. *With other operations, such as the maze procedure.

\section{SPA Score and ICU LOS}

The distribution of SPA scores and sex, age, and selected comorbidities is shown in Table 2. The proportion of patients assigned SPA scores of $1 \mathrm{~A}, 1 \mathrm{~B}, 2 \mathrm{~A}, 2 \mathrm{~B}$, and 3 was $32.7 \%, 13.7 \%, 25.2 \%, 25.6 \%$, and $2.7 \%$, respectively. There were significantly fewer female patients who had scores of SPA 1A or 3. Patients with scores of SPA 3 were significantly younger than those in the other SPA groups, had significantly lower preoperative left ventricular ejection fraction, and also had a significantly higher incidence of end-stage renal disease $(12.1 \%)$ compared with patients with SPA scores of $1 \mathrm{~B}(2.9 \%)$ and $2 \mathrm{~B}(6.7 \%)$. There was no difference in the incidence of preexisting diabetes between the groups. 


\section{Length of Intensive Care Unit Stay / days Comparison of SPA score with other scores \\ SPA score}
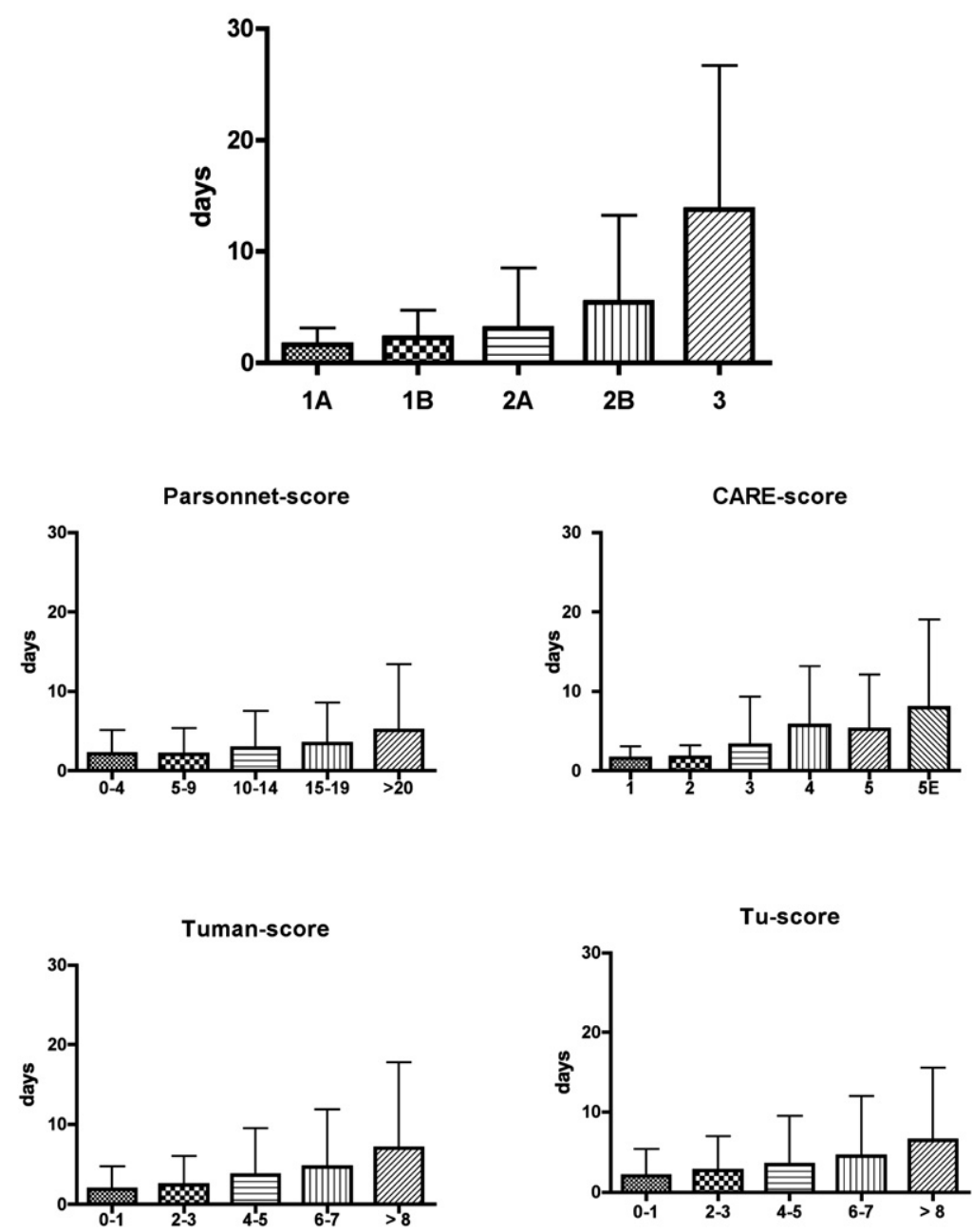

FIGURE 1. Intensive care unit length of stay after cardiac surgery comparing the Surgical Procedure Assessment (SPA) score with the Parsonnet, Cardiac Anesthesia Risk Evaluation (CARE), Tuman, and Tu scores (means \pm standard deviations).

There was an exponential and statistically significant increase in ICU LOS with increasing SPA scores (Figure 1, A). The mean ICU LOS between SPA scores 1A, 1B, 2A, $2 \mathrm{~B}$, and 3 increased from $1.6 \pm 1.5$ days to $2.3 \pm 2.5$, $3.1 \pm 5.4,5.4 \pm 7.8$, and $13.8 \pm 12.9$ days, respectively $(P<.01$, Cuzick's nonparametric test for trend). Comparable graphs for the Parsonnet, Tuman, Tu, and CARE scores are shown in Figure 1, $B$ to $E$. Hospital LOS, mortality, and charges also increased significantly with each incremental increase in SPA score (Figure 2).

\section{Prediction of ICU LOS}

We developed regression models to assess the ability of the SPA score to explain the variability of ICU and hospital LOS, mortality, and hospital charges compared with the Parsonnet, Tuman, Tu, or CARE scores. After $\log$ transformation of LOS and charges to achieve a Gaussian distribution, ICU and hospital LOS and charges were modeled with linear regression by using a stepwise procedure with backward elimination. A logistic regression model was used to assess the ability of the scores to explain the variability of mortality. All logistic models fit the data well with the Hosmer and Lemeshow goodness-of-fit test. Receiver operating characteristic curve analyses suggested that the SPA score provides the largest area under the curve (ie, 0.781), whereas the 4 other scoring systems have areas under the curve ranging from 0.725 to 0.774 (Table 3). The SPA score performed best at explaining the variability of ICU LOS, mortality, and hospital charges, and the CARE score 


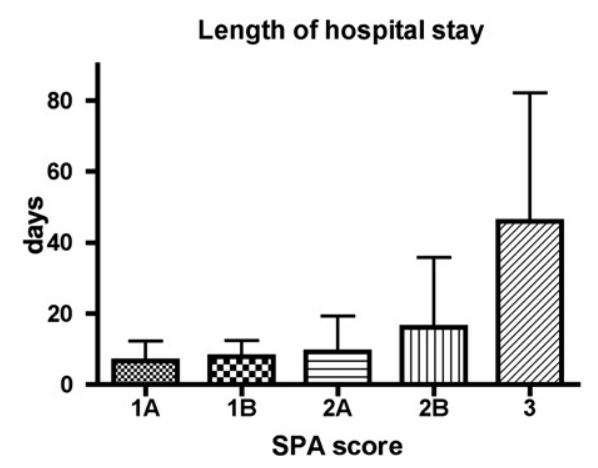

Panel A: Length of hospital stay after cardiac surgery increases incrementally with increasing Surgical Procedure Assessment (SPA)

Mortality

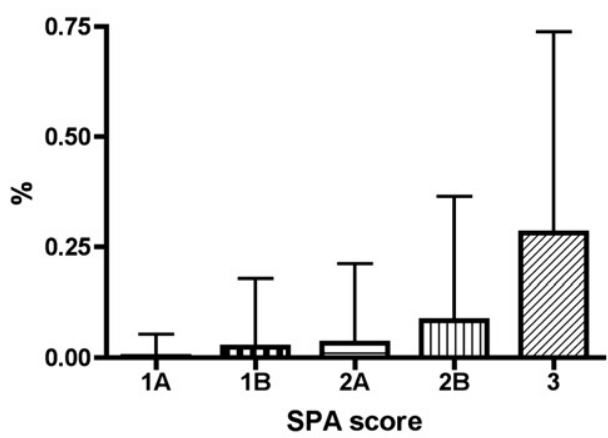

Panel B: Hospital mortality (\%) increases incrementally with increasing Surgical Procedure Assessment (SPA)

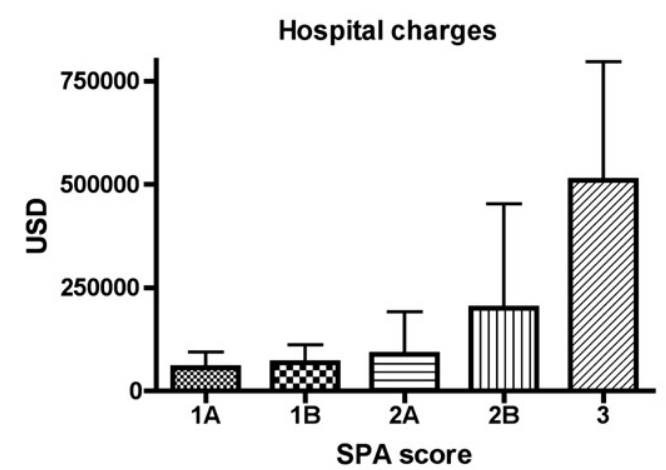

Panel C: Hospital charges (in US Dollars) increase incrementally with increasing Surgical Procedure Assessment (SPA)

FIGURE 2. The Surgical Procedure Assessment (SPA) score and length of hospital stay (A), mortality (B), and hospital charges (C, in US dollars [USD]; means \pm standard deviations).

performed best at explaining the variability in hospital LOS (Table 4).

\section{Identification of Fast-Track Patients}

Of the 393 patients with an SPA score of 1A, 306 $(77.9 \%)$ had an ICU LOS of less than 48 hours, and $182(46.3 \%)$ had an ICU LOS of less than 24 hours. In contrast, $393(49 \%)$ of 808 patients with an SPA score of greater than 1A had an ICU LOS of more than 48 hours, and $184(22.8 \%)$ had an ICU LOS less than 24 hours.

An SPA score of $1 \mathrm{~A}$ was better able to predict a short ICU stay, whether less than 48 hours or less than 24 hours, than a Parsonnet score of less than 5, a Tuman score of less than 2, a Tu score of less than 2, or a CARE score of 1 . The sensitivities, specificities, relative risks, odds ratios, positive and negative predictive values, and likelihood ratios are depicted in Table 5. 
TABLE 3. Logistic regression model of in-hospital mortality for scoring system comparison

\begin{tabular}{lccccc}
\hline & & \multicolumn{4}{c}{ Logistic regression model } \\
\cline { 3 - 6 } Scoring & ROC & & & Wald & $\boldsymbol{P}$ value, \\
system & curve AUC & Coefficient & $\boldsymbol{P}$ value & $\boldsymbol{\chi}^{\mathbf{2}}$ test & HL test \\
\hline SPA & 0.781 & 1.061 & $<.0001$ & 38.9 & 0.32 \\
Parsonnet & 0.725 & 0.074 & $<.0001$ & 45.9 & 0.46 \\
CARE & 0.766 & 0.768 & $<.0001$ & 27.4 & 0.19 \\
Tuman & 0.746 & 0.317 & $<.0001$ & 37.8 & 0.20 \\
Tu & 0.744 & -0.464 & .08 & 3.0 & 0.79 \\
\hline
\end{tabular}

ROC curve AUC, Area under the curve of the receiver operator curve of the scoring system to predict in-hospital mortality; HL test, Hosmer and Lemeshow goodness-offit test $(P<.05$ rejects $\mathrm{H} 0$ that the model fits the data well); SPA, Surgical Procedure Assessment; CARE, Cardiac Anesthesia Risk Evaluation.

\section{Identification of Prolonged-Stay Patients}

Of the 340 patients with an SPA score of $2 \mathrm{~B}$ or 3,273 $(80.3 \%)$ had an ICU LOS of more than 7 days, and 181 $(53.2 \%)$ had an ICU LOS of more than 10 days. In contrast, $366(42.5 \%)$ of 861 patients with an SPA score of greater than $2 \mathrm{~B}$ had an ICU LOS of more than 7 days, and 148 $(17.2 \%)$ had an ICU LOS of more than 10 days.

An SPA score of $2 \mathrm{~A}$ or 3 was better able to predict prolonged ICU stay, whether more than 7 days or more than 10 days, than a Parsonnet score of greater than 15, a Tuman score of greater than 6 , a Tu score of greater than 6 , or a CARE score of 4,5 , or $5 \mathrm{E}$. The sensitivities, specificities, relative risks, odds ratios, positive and negative predictive values, and likelihood ratios are depicted in Table 6.

\section{DISCUSSION}

We have demonstrated that the SPA score, a simple, intuitive preoperative assignment of risk, can predict ICU LOS after cardiac surgery. It does so as well or better than the Parsonnet, Tuman, Tu, or CARE scores. Moreover, it is better able to discriminate between fast-track (ICU LOS $<48$ hours) and prolonged-stay ( $>7$ days) patients. The SPA score also correlates with hospital LOS, charges, and inhospital mortality.

The ability to preoperatively predict short versus prolonged LOS greatly facilitates ICU throughput and allocation of ICU resources and improves the efficiency of a cardiac surgical service. Today, the lack of available
ICU beds is all too often the rate-limiting step in reaching target numbers of cardiac surgical procedures. There are numerous reasons for this problem. Many hospitals were built in an age when cardiac surgical patients were younger and healthier and created a ratio between ICU and general beds lower than currently required. With the ascent of percutaneous coronary intervention in the last 2 decades, patients coming to cardiac surgery have become progressively older and have greater comorbidity. ${ }^{10,11}$ Complex surgical procedures are performed on patients at the extremes of life or who have advanced, decompensated congestive heart failure. ${ }^{12}$ Even if these patients comprise a relatively small proportion of the cardiac surgical mix, they consume an inordinate amount of ICU resources. For example, in our study less than $10 \%$ of patients had a prolonged stay in the ICU, but they used nearly $50 \%$ of all ICU bed days.

The institution of fast-track protocols has allowed us to minimize the time cardiac surgical patients remain in the ICU or, in some institutions, to bypass the ICU altogether. ${ }^{13,14}$ However, there is a lack of reliable tools to identify potential candidates for fast-track protocols before surgical intervention. The most well-established risk assessment score is the Parsonnet score, ${ }^{5}$ a 22 -factor scale developed in 1989 to stratify cardiac surgical procedures into 5 levels of mortality risk. In 1992, Tuman and colleagues ${ }^{6}$ developed a 14 -factor model to stratify the risk of serious morbidity after cardiac surgery based on 11 variables. In 1995, Tu and associates ${ }^{7}$ presented a similar 14point risk index for mortality, ICU, and hospital LOS. It is a simple risk index based on 6 variables, 3 related to the type of operation and 1 related to ventricular function (but not patient comorbidity), that are used to predict mortality and ICU and hospital LOS. Finally, in 2001, a cardiac anesthesia risk evaluation (CARE) score was devised. ${ }^{8}$ Based on "controlled" or "uncontrolled" comorbidity, surgical complexity, and urgency, it is used to assess the risk of postoperative morbidity and mortality.

We found that the SPA score is superior to these systems in being able to preoperatively discriminate between the 2 groups of patients (ie, fast track and prolonged stay) that have the largest effect on ICU resource use and efficiency.

TABLE 4. Linear regression models of ICU LOS, hospital LOS, and hospital charges for scoring system comparison

\begin{tabular}{|c|c|c|c|c|c|c|c|c|c|}
\hline \multirow[b]{2}{*}{ Scoring system } & \multicolumn{3}{|c|}{ ICU LOS } & \multicolumn{3}{|c|}{ Hospital LOS } & \multicolumn{3}{|c|}{ Hospital charges } \\
\hline & Coefficient & $P$ value & $R^{2}$ & Coefficient & $P$ value & $R^{2}$ & Coefficient & $P$ value & $R^{2}$ \\
\hline SPA & 0.321 & $<.0001$ & 0.188 & 0.292 & $<.0001$ & 0.197 & 0.320 & $<.0001$ & 0.281 \\
\hline Parsonnet & 0.028 & $<.0001$ & 0.112 & 0.022 & $<.0001$ & 0.093 & 0.024 & $<.0001$ & 0.139 \\
\hline CARE & 0.273 & $<.0001$ & 0.156 & 0.285 & $<.0001$ & 0.203 & 0.290 & $<.0001$ & 0.261 \\
\hline Tuman & 0.133 & $<.0001$ & 0.142 & 0.106 & $<.0001$ & 0.117 & 0.111 & $<.0001$ & 0.157 \\
\hline $\mathrm{Tu}$ & 0.015 & .4 & 0.055 & 0.001 & .566 & 0.030 & -0.011 & .389 & 0.02 \\
\hline
\end{tabular}

LOS and charges were log-transformed before modeling. ICU, Intensive care unit; LOS, length of stay; SPA, Surgical Procedure Assessment; CARE, Cardiac Anesthesia Risk Evaluation. 
TABLE 5. The ability of the SPA score to identify 2 groups of fast-track patients compared with the PS, CARE, Tuman, and Tu scores

\begin{tabular}{lcccrc}
\hline \multicolumn{1}{c}{ Part A: $\mathbf{0 - 2 4} \mathbf{h}^{*}$} & SPA $=\mathbf{1 A}$ & $\mathbf{P S}<\mathbf{5}$ & CARE $=\mathbf{1}$ & Tuman $<\mathbf{2}$ & Tu $<\mathbf{2}$ \\
\hline Relative risk & $1.97(1.68-2.30)$ & $1.84(1.41-2.41)$ & $2.5(1.98-3.39)$ & $2.03(1.66-2.49)$ & $2.09(1.63-2.67)$ \\
Odds ratio & $2.9(2.26-3.79)$ & $2.08(1.50-2.88)$ & $3.13(2.25-4.34)$ & $2.61(1.98-3.45)$ & $2.48(1.82-3.38)$ \\
Sensitivity & $0.46(0.41-0.51)$ & $0.44(0.37-0.52)$ & $0.53(0.45-0.60)$ & $0.47(0.41-0.53)$ & $0.47(0.40-0.54)$ \\
Specificity & $0.77(0.74-0.80)$ & $0.72(0.69-0.745)$ & $0.73(0.71-0.76)$ & $0.745(0.72-0.77)$ & $0.73(0.70-0.76)$ \\
Positive predictive value & $0.49(0.44-0.55)$ & $0.21(0.18-0.267)$ & $0.25(0.21-30)$ & $0.36(0.31-0.41)$ & $0.27(0.22-0.31)$ \\
Negative predictive value & $0.75(0.71-0.78)$ & $0.88(0.86-0.90)$ & $0.90(0.88-0.92)$ & $0.82(0.79-0.85)$ & $0.87(0.85-0.89)$ \\
Pearson $\chi^{2}$ test & 69.1 & 49.7 & 20.1 & 34.5 & 47.3 \\
\hline
\end{tabular}

\begin{tabular}{lccccc}
\multicolumn{1}{c}{ Part B: $\mathbf{0 - 4 8} \mathbf{~ h} \dagger$} & SPA $=\mathbf{1 A}$ & PS $<\mathbf{5}$ & CARE $=\mathbf{1}$ & Tuman $<\mathbf{2}$ & Tu $<\mathbf{2}$ \\
\hline Relative risk & $2.53(2.05-3.11)$ & $1.85(1.37-2.52)$ & $2.68(1.90-3.78)$ & $2.22(1.73-2.84)$ & $1.93(1.45-2.58)$ \\
Odds ratio & $3.71(2.82-4.89)$ & $2.05(1.44-2.90)$ & $3.09(2.11-4.54)$ & $2.74(2.03-3.71)$ & $2.18(1.56-3.05)$ \\
Sensitivity & $0.78(0.73-0.82)$ & $0.72(0.65-0.79)$ & $0.79(0.72-0.85)$ & $0.76(0.70-0.80)$ & $0.73(0.66-0.79)$ \\
Specificity & $0.51(0.48-0.55)$ & $0.44(0.41-0.47)$ & $0.45(0.42-0.48)$ & $0.47(0.44-0.50)$ & $0.45(0.42-0.48)$ \\
Positive predictive value & $0.44(0.40-0.48)$ & $0.18(0.16-0.22)$ & $0.20(0.17-0.23)$ & $0.30(0.27-0.34)$ & $0.21(0.18-0.24)$ \\
Negative predictive value & $0.83(0.79-0.86)$ & $0.90(0.87-0.93)$ & $0.93(0.90-0.95)$ & $0.86(0.83-0.89)$ & $0.89(0.86-0.92)$ \\
Pearson $\chi^{2}$ test & 92.8 & 35.9 & 16.6 & 21.7 & 44.7 \\
\hline
\end{tabular}

*The relative risks, odds ratios, sensitivities, specificities, positive and negative predictive values, and Pearson $\chi^{2}$ test results of an SPA score of $1 \mathrm{~A}$ to predict patients who require 0 to 24 hours in the intensive care unit compared with the other scoring systems are shown, with $95 \%$ confidence intervals in parentheses. $\nmid$ The relative risks, odds ratios, sensitivities, specificities, positive and negative predictive values, and Pearson $\chi^{2}$ test results of an SPA score of $1 \mathrm{~A}$ to predict patients who require 0 to 48 hours in the intensive care unit compared with the other scoring systems are shown, with 95\% confidence intervals shown in parentheses. SPA, Surgical Procedure Assessment; PS, Parsonnet; CARE, Cardiac Anesthesia Risk Evaluation.

At Columbia University Medical Center, the SPA score has been in routine daily use as an integral part of the published cardiac operating room schedule for more than 7 years. The SPA score is assigned in the cardiac surgical office during the patient's preoperative visit, and all members of the care team are familiar with it, including cardiac surgeons, cardiac anesthesiologists, scheduling administrators, triage coordinators, operating room and ICU nursing staff, and the ICU medical team. The SPA score allows us to pre- dict the effect of a given surgical schedule on the ICU on any given day. For example, if 5 of 8 scheduled patients have SPA scores of 1A, we can anticipate a strong likelihood that those 5 ICU beds will again become available within 24 hours. Conversely, if only 2 patients have SPA scores of $1 \mathrm{~A}$, it forewarns us that proactive triage (eg, assigning some patients to the surgical ICU) will be required to accommodate all ICU patients in the cardiothoracic ICU the next day.

TABLE 6. The ability of the SPA score to identify 2 groups of prolonged-stay patients compared with the PS, CARE, Tuman, and Tu scores

\begin{tabular}{lccccc}
\hline \multicolumn{1}{c}{ Part A: $>$ 7 d* } & SPA 2B or 3 & PS $\geq \mathbf{1 5}$ & CARE $\geq \mathbf{4}$ & Tuman $\geq \mathbf{6}$ & Tu $\geq \mathbf{6}$ \\
\hline Relative risk & $3.58(2.81-4.57)$ & $1.88(1.64-2.15)$ & $2.42(1.98-2.98)$ & $2.73(2.14-3.48)$ & $2.65(2.09-3.37)$ \\
Odds ratio & $5.51(4.08-7.43)$ & $3.17(2.49-4.02)$ & $3.44(2.625-4.507)$ & $3.61(2.680-4.875)$ & $3.52(2.618-4.725)$ \\
Sensitivity & $0.80(0.76-0.84)$ & $0.68(0.64-0.72)$ & $0.73(0.69-0.78)$ & $0.76(0.70-0.80)$ & $0.75(0.69-0.79)$ \\
Specificity & $0.57(0.54-0.61)$ & $0.59(0.56-0.64)$ & $0.55(0.52-0.59)$ & $0.54(0.51-0.57)$ & $0.54(0.51-0.57)$ \\
Positive predictive value & $0.43(0.39-0.47)$ & $0.59(0.55-0.63)$ & $0.41(0.38-0.45)$ & $0.33(0.30-0.38)$ & $0.34(0.31-0.38)$ \\
Negative predictive value & $0.88(0.85-0.91)$ & $0.68(0.64-0.72)$ & $0.83(0.79-0.86)$ & $0.88(0.85-0.90)$ & $0.87(0.84-0.89)$ \\
Pearson $\chi^{2}$ test & 139.8 & 84.6 & 92.9 & 74.5 & 76.0 \\
\hline
\end{tabular}

\begin{tabular}{lccccc} 
Pearson $\chi^{2}$ test & 139.8 & 84.6 & 92.9 & 74.5 & 76.0 \\
\multicolumn{1}{c}{ Part B: $>\mathbf{1 0} \mathbf{d} \dagger$} & SPA 2B or 3 & PS $\geq \mathbf{1 5}$ & CARE $\geq \mathbf{4}$ & Tuman $\geq \mathbf{6}$ & Tu $\geq \mathbf{6}$ \\
\hline Relative risk & $3.02(2.54-3.58)$ & $1.66(1.48-1.86)$ & $2.79(2.37-3.28)$ & $2.07(1.71-2.52)$ & $1.87(1.53-2.27)$ \\
Odds ratio & $5.48(4.16-7.24)$ & $2.91(2.23-3.79)$ & $5.08(3.87-6.68)$ & $2.74(2.07-3.623)$ & $2.37(1.79-3.13)$ \\
Sensitivity & $0.53(0.48-0.59)$ & $0.385(0.345-0.427)$ & $0.513(0.46-0.57)$ & $0.44(0.38-0.49)$ & $0.41(0.36-0.47)$ \\
Specificity & $0.83(0.80-0.85)$ & $0.82(0.79-0.85)$ & $0.83(0.80-0.84)$ & $0.78(0.75-0.80)$ & $0.77(0.74-0.79)$ \\
Positive predictive value & $0.55(0.49-0.60)$ & $0.65(0.599-0.70)$ & $0.56(0.51-0.62)$ & $0.38(0.33-0.44)$ & $0.37(0.32-0.42)$ \\
Negative predictive value & $0.82(0.79-0.84)$ & $0.61(0.57-0.64)$ & $0.79(0.77-0.82)$ & $0.82(0.79-0.84)$ & $0.80(0.77-0.83)$ \\
Pearson $\chi^{2}$ test & 159.2 & 147.6 & 65.0 & 37.7 & 112.4 \\
\hline
\end{tabular}

*The relative risks, odds ratios, sensitivities, specificities, positive and negative predictive values, and Pearson $\chi^{2}$ test results of an SPA score of $2 \mathrm{~B}$ or 3 to predict patients needing greater than 7 days of intensive care unit care compared with the other scoring systems are shown, with $95 \%$ confidence intervals in parentheses. $\dagger$ The relative risks, odds ratios, sensitivities, specificities, positive and negative predictive values, and Pearson $\chi^{2}$ test results of an SPA score of $2 \mathrm{~B}$ or 3 to predict patients needing greater than 10 days of intensive care unit care compared with the other scoring systems are shown, with 95\% confidence intervals shown in parentheses. SPA, Surgical Procedure Assessment; PS, Parsonnet; CARE, Cardiac Anesthesia Risk Evaluation. 
There are a number of limitations to this study in general and the SPA score in particular. We did not include the EuroSCORE ${ }^{15}$ in the array of systems we compared because it was not in use at our institution at the time of this study, although, like the Parsonnet Score, it is a complex scale that was designed to predict operative mortality and not ICU LOS. The SPA score itself is simple in concept and practice, but its value is quite dependent on the consistency and accuracy with which it is applied. The system works best when the cardiac surgeon takes responsibility for quality control of the assignment or surgical complexity and comorbidity. Obviously no preoperative scoring system takes into account complications that occur during or after surgical intervention and that provide an important effect on ultimate ICU LOS. In this sense a preoperative scoring assignment, such as the SPA score, is only one step in what should be a multimodal approach to ICU triage. For example, if the surgical scheduler can ensure that a substantial number of cases each day are scored as SPA 1A, with a predictably short ICU LOS, this could moderate the effect of patients with high morbidities undergoing complex operations who might occupy ICU beds for a prolonged period. Scheduling more complex cases with higher SPA scores toward the end of the week maximizes the use of the ICU over the weekend to facilitate bed availability for the next week.

In conclusion, we have demonstrated that preoperative assignment of the SPA score reliably helps predict ICU LOS after cardiac surgery and is able to discriminate between fast-track (ICU LOS $<48$ hours) and prolonged-stay ( $>7$ days) patients. The SPA score also correlates with hospital LOS, charges, and in-hospital mortality. In sum, we have found that the SPA score is an excellent tool to achieve a more even, rational, and efficient use of the ICU and improve communication between all caregivers.

\section{References}

1. Ferguson TB Jr, Hammill BG, Peterson ED, DeLong ER, Grover FL. A decade of change-risk profiles and outcomes for isolated coronary artery bypass grafting procedures, 1990-1999: a report from the STS National Database Committee and the Duke Clinical Research Institute. Society of Thoracic Surgeons. Ann Thorac Surg. 2002;73:480-90

2. Williams MR, Wellner RB, Hartnett EA, Thornton B, Kavarana MN, Mahapatra R, et al. Long-term survival and quality of life in cardiac surgical patients with prolonged intensive care unit length of stay. Ann Thorac Surg. 2002; 73:1472-8.

3. Mazer CD, Byrick RJ, Sibbald WJ, Chovaz PM, Goodman SJ, Girotti MJ, et al. Postoperative utilization of critical care services by cardiac surgery: a multicenter study in the Canadian healthcare system. Crit Care Med. 1993;21:851-9.

4. Zenati M, Cohen HA, Holubkov R, Smith AJ, Boujoukos AJ, Caldwell J, et al. Preoperative risk models for minimally invasive coronary bypass: a preliminary study. J Thorac Cardiovasc Surg. 1998;116:584-9.

5. Parsonnet V, Dean D, Bernstein AD. A method of uniform stratification of risk for evaluating the results of surgery in acquired adult heart disease. Circulation. 1989;79(suppl I):I3-12.

6. Tuman KJ, McCarthy RJ, March RJ, Najafi H, Ivankovich AD. Morbidity and duration of ICU stay after cardiac surgery. A model for preoperative risk assessment. Chest. 1992;102:36-44.

7. Tu JV, Jaglal SB, Naylor CD. Multicenter validation of a risk index for mortality, intensive care unit stay, and overall hospital length of stay after cardiac surgery. Steering Committee of the Provincial Adult Cardiac Care Network of Ontario. Circulation. 1995;91:677-84.

8. Dupuis JY, Wang F, Nathan H, Lam M, Grimes S, Bourke M. The cardiac anesthesia risk evaluation score: a clinically useful predictor of mortality and morbidity after cardiac surgery. Anesthesiology. 2001;94:194-204.

9. Cuzick JA. Wilcoxon-type test for trend. Stat Med. 1985;4:87-90.

10. Ulrich MR, Brock DM, Ziskind AA. Analysis of trends in coronary artery bypass grafting and percutaneous coronary intervention rates in Washington state from 1987 to 2001. Am J Cardiol. 2003;92:836-9.

11. Bonaros N, Hennerbichler D, Friedrich G, Kocher A, Pachinger O, Laufer G, et al. Increased mortality and perioperative complications in patients with previous elective percutaneous coronary interventions undergoing coronary artery bypass surgery. J Thorac Cardiovasc Surg. 2009;137:846-52.

12. Movahed MR, Ramaraj R, Jamal MM, Hashemzadeh M. Nationwide trends in the utilisation of percutaneous coronary intervention (PCI) in the United States of America based on gender and ethnicities. EuroIntervention. 2009;5:343-8.

13. Pande RU, Nader ND, Donias HW, D'Ancona G, Karamanoukian HL. Fasttracking cardiac surgery. Heart Surg Forum. 2003;6:244-8.

14. Lake CL. Fast tracking in paediatric cardiac anaesthesia: an update. Ann Card Anaesth. 2002;5:203-8.

15. Roques F, Michel P, Goldstone AR, Nashef SA. The logistic EuroSCORE. Eur Heart J. 2003;24:881-2. 